\title{
Hydrogel-Based Nanocomposites and Mesenchymal Stem Cells: A Promising Synergistic Strategy for Neurodegenerative Disorders Therapy
}

\author{
Diego Albani, ${ }^{1}$ Antonio Gloria, ${ }^{2}$ Carmen Giordano, ${ }^{3}$ Serena Rodilossi, ${ }^{1}$ Teresa Russo, ${ }^{2}$ \\ Ugo D'Amora, ${ }^{2}$ Marta Tunesi, ${ }^{3}$ Alberto Cigada, ${ }^{3}$ Luigi Ambrosio, ${ }^{2}$ and Gianluigi Forloni ${ }^{1}$ \\ ${ }^{1}$ IRCCS- "Mario Negri" Institute for Pharmacological Research, Via La Masa 19, 20156 Milan, Italy \\ ${ }^{2}$ Institute of Composite and Biomedical Materials, National Research Council of Italy, P.le Tecchio 80, 80125 Naples, Italy \\ ${ }^{3}$ Department of Chemistry, Materials and Chemical Engineering "G. Natta," Politecnico of Milan and \\ INSTM Consortium Research Unit, 20131 Milan, Italy \\ Correspondence should be addressed to Diego Albani; diego.albani@marionegri.it
}

Received 4 October 2013; Accepted 26 November 2013

Academic Editors: R. Adhikari and L. Visai

Copyright (c) 2013 Diego Albani et al. This is an open access article distributed under the Creative Commons Attribution License, which permits unrestricted use, distribution, and reproduction in any medium, provided the original work is properly cited.

\begin{abstract}
Hydrogel-based materials are widely employed in the biomedical field. With regard to central nervous system (CNS) neurodegenerative disorders, the design of injectable nanocomposite hydrogels for in situ drug or cell release represents an interesting and minimally invasive solution that might play a key role in the development of successful treatments. In particular, biocompatible and biodegradable hydrogels can be designed as specific injectable tools and loaded with nanoparticles (NPs), to improve and to tailor their viscoelastic properties upon injection and release profile. An intriguing application is hydrogel loading with mesenchymal stem cells (MSCs) that are a very promising therapeutic tool for neurodegenerative or traumatic disorders of the CNS. This multidisciplinary review will focus on the basic concepts to design acellular and cell-loaded materials with specific and tunable rheological and functional properties. The use of hydrogel-based nanocomposites and mesenchymal stem cells as a synergistic strategy for nervous tissue applications will be then discussed.
\end{abstract}

\section{Introduction}

Mesenchymal stem cells (MSCs) are a very promising therapeutic tool for neurodegenerative or traumatic disorders affecting the central nervous system (CNS). For instance, they have been considered for regenerative purposes for Parkinson's disease (PD), multiple sclerosis (MS), and amyotrophic lateral sclerosis (ALS), as well as traumatic brain injury (TBI), spinal cord injury (SCI), or nerve repair [1-3].

Polymers represent an attractive platform for MSCs applications thanks to their tunable properties and high versatility [4-7]. In particular, although their design and optimization set a great challenge, injectable nanocomposite hydrogels (i.e., hydrogels loaded with nanoparticles, NPs) for in situ cell or drug release represent a minimally invasive solution to enhance the effectiveness of potential therapeutic strategies and might be usefully exploited for the treatment of severe neurodegenerative disorders like PD and Alzheimer's disease (AD) $[4,5]$. In this scenario, coupling MSCs and nanostructured hydrogels might therefore help to take advantage and translate their therapeutic potential into clinical approaches.

In order to strongly support this conclusion, the rheological and functional properties of injectable hydrogels will be preliminarILy discussed; then current applications of nanocomposite hydrogels in MSC-based therapy for nervous tissue applications will be presented, as well as describing the current research carried out by the authors in the field of CNS neurodegenerative disorders, like PD and AD.

\section{Injectable Hydrogel-Based Nanocomposites: Rheological and Functional Features}

Several injectable formulations of gels and hydrogels have been developed for a wide range of biomedical applications 
[8-16]. In particular, hydrogels have proved to be an optimal tool for cell-based strategies, even though rheological properties of acellular and cell-laden gels might vary considerably [17-19]. From a materials science point of view, rheological and injectability measurements, together with confined compression tests, may provide important information on their functional properties among which the functional injectability plays a crucial role [20].

The injection of a gel could negatively affect its rheological behaviour and viscoelastic properties (storage and loss moduli, $G^{\prime}$ and $G^{\prime \prime}$, respectively). In fact, the injection through clinical catheters could cause a partial or total disruption of the polymeric network, thus leading to a decrease of the storage modulus and an alteration of the gel-like behaviour. To improve the viscoelastic properties without altering the gel-like behaviour of both acellular or cell-laden hydrogels [20], NPs are often included as a reinforcement. Accordingly, after the injection, $G^{\prime}$ and $G^{\prime \prime}$ may decrease, but the inclusion of NPs may provide values of both dynamic moduli suitable for the specific application [21-25]. The amount of NPs represents a critical factor, since they provide a resistance of the material to flow under shear. The storage modulus and the viscosity normally increase with NP concentration, but beyond a threshold concentration $\mathrm{G}^{\prime}$ dramatically decreases, even though NP concentration is further increased, and during the injection the NPs act as "weak points" instead of reinforcement. For this reason, the optimization of injectable nanocomposites as well as the prediction of their rheological behaviour represents a great challenge that may be overcome by integrating experimental tests and mathematical models [23]. A very recent example of nanocomposite hydrogels in the specific field of this review is from Koudehi et al. [26]. They developed a nanobioglass conduit for peripheral nerve regeneration by coupling bioglass NPs with gelatin and obtaining a microporous nanocomposite having the pore size of $10-40 \mu \mathrm{m}$, good biocompatibility, and good nerve regeneration in a rat model.

With regard to cell-laden nanocomposite gels, small amplitude oscillatory shear tests may be performed at different time points after cell seeding to evaluate $G^{\prime}$ and $G^{\prime \prime}$ as a function of frequency, in order to understand the effect of cell behaviour on the viscoelastic properties of the material and to optimize the cell density [20]. At each time point, it is expected that the values of the loss tangent $\left(G^{\prime \prime} / G^{\prime}\right)$ are greater than those of the corresponding acellular gels and they increase with culture time.

Viscosity as a function of shear rate may be assessed by steady shear tests; however, the strains induced by oscillatory rheometry are usually smaller than those achieved during extrusion/injection-based measurements and hence less likely to alter the structure of the material $[27,28]$. In addition, by performing only small amplitude oscillatory shear tests and steady shear measurements it might be difficult to get shear strains and rates similar to those occurring in clinical practice, where the materials are applied by injectionlike methods involving the use of a syringe with a suitable needle. To measure the flow behaviour while simulating the clinical practice, an appropriate injection-based experimental setup is required. For instance, the syringe (equipped with a needle suitable for the final application) may be filled with the material and mounted on a testing machine. By driving the syringe piston at a constant and fixed speed (thus controlling the injection rate and the apparent shear rate in the needle), the material is injected through the needle and the load applied to the piston may be measured by a suitably calibrated load cell. To estimate the friction between the syringe walls and the piston, an empty syringe should be also tested, while to assess the effect of the cells on the flow behaviour over time, the acellular nanocomposite gels should be compared to the cell-laden ones at different time points after seeding. Finally, the results from these injectability measurements may be used to evaluate some functional features and rheological information by applying the basic principles of capillary extrusion rheometry.

Due to the biphasic nature of hydrogel-based materials, confined compression stress-relaxation tests may be also performed on both acellular and cell-laden nanocomposites to evaluate functional parameters, such as the zero-strain compressive modulus, the zero-strain permeability, the nonlinear stiffening coefficient, and the nonlinear permeability coefficient that is a measure of the sensitivity to the deformation. As reported in $[27,28]$, different equations may be considered to study the case of uniaxial confined compression, starting from the constitutive law for the extra-stress tensor to the relationship between the hydraulic permeability and the axial deformation to obtain a one-dimensional nonlinear partial differential equation.

In terms of transport properties, permeability plays a key role, since the fluid flow through the porous solid matrix controls the deformational process [29-31]. As reported by Ateshian et al. [29] and Williamson et al. [30], the straindependent permeability may be properly assessed by fitting the experimental data from the confined compression tests with a nonlinear biphasic model.

In conclusion, all the results from rheological analyses, injectability measurements, and, eventually, transport properties should be taken into account to tailor and optimize the functional features of both acellular and cell-laden gels for tissue engineering applications. Focusing on the CNS, their integration with biological expertise may be fully exploited to pursue innovative approaches involving the synergistic contribution of MSCs and hydrogel-based nanocomposites.

\section{Hydrogel and MSCs as a Synergistic Strategy for Nervous Tissue Applications}

Over the past few years, the basic approach of using hydrogels for regenerative or neuroprotective strategies in the nervous tissue has been explored. For instance, Freudenberg et al. [32] developed biohybrid hydrogel platforms based on covalently cross-linked heparin and star-shaped poly(ethylene glycols) (star-PEGs) for potential use in cell replacement therapies for neurodegenerative diseases. They kept the amount of heparin constant and analyzed the effect of the cross-linking degree on the mesh size, swelling, and elastic modulus; furthermore, they demonstrated the impact of biomolecular and mechanical cues on neural stem cells and primary nerve cells. 
PEG hydrogels coupled with soluble factors were investigated by Mooney et al. [33], who evidenced how the hydrogel environment may influence neural cell composition and described soluble factors that may be useful in generating neuronal-enriched populations within the specific hydrogel environment.

To obtain successful biomaterial-cell interactions, the modification of synthetic hydrogels with biologically active molecules has become an increasingly important route. For instance, Zhou et al. [34] used the monomer 2-methacryloxyethyl trimethylammonium chloride (MAETAC) to provide tethered neurotransmitter acetylcholine-like functionality with a complete 2 -acetoxy-N,N,N-trimethylethanaminium segment. The results from this study showed that MAETAC could promote neuronal cell attachment and differentiation in a concentration-dependent manner.

The expertise gained thanks to the above mentioned scenario paves the way to an intriguing approach: the use of MSCs in cell/material biohybrid constructs for CNS applications. MSCs may be used undifferentiated as a reservoir of trophic factors or they may be differentiated towards a neuronal phenotype for regenerative or replacement therapies. While for the first approach a systemic injection into the blood stream might be suitable [35], for the second one the placement of MSCs at or within the lesion site might benefit from the presence of a hydrogel aiming at ameliorating cell survival and therapeutic performance. For instance, Ghoreishian et al. [36] embedded undifferentiated MSCs from the autogenous adipose tissues of mongrel dogs into an alginate hydrogel and placed the construct into an expanded poly(tetrafluoroethylene) tube to repair a facial nerve lesion. After 12 weeks, an organized neural tissue was formed within the tubes, having a decreased diameter of $29 \%$ in the experimental group. Neurofilament-positive axon counts were $67 \%$ of normal values and there was no significant difference between the groups in relation to the histomorphometric parameters; furthermore, nerve conduction velocity was significantly greater in the experimental than in the control group. Despite some limitations, these results suggest that a similar approach exploiting different biocompatible materials is promising.

Kubinovà et al. [37] tried a different approach using a methacrylate hydrogel scaffold to support MSC proliferation. They modified poly(2-hydroxyethyl methacrylate) (PHEMA) with cholesterol to get highly superporous hydrogels that may serve as a permissive scaffold for the treatment of spinal cord injury (SCI). In vitro the hydrogels supported the adhesion and proliferation of rat MSCs, while in vivo they displayed bioadhesive properties and were able to bridge a spinal cord lesion in a rat model. For the same application, other authors [38] proposed an injectable hydrogel with a controlled nanostructure, together with a new protocol for building three-dimensional (3D) biohybrid cell/hydrogel constructs. Their matrix was tested with glial populations, primary astrocytes, and MSCs and the results indicated that the cells survived the period of latency within the hydrogel.

The concept of developing a 3D scaffold to support MSC proliferation or differentiation was also pursued by $\mathrm{Gu}$ et al. [39]. They developed macroporous, cellulosic hydrogels to induce the neuronal differentiation of human MSCs (hMSCs). After 7 days the number of hMSCs in the scaffolds increased by more than 14-fold, while after 14 days of induction they could completely differentiate into neurons and glial cells. These results appear very promising, but they might be further improved by the nanostructuration and/or functionalization of the hydrogels. A similar approach was also described by Wang et al. [40]. In this case, the hydrogels were composed of gelatin-hydroxyphenylpropionic acid (Gtn-HPA) conjugate and synthesized by the oxidative coupling of HPA moieties, catalyzed by hydrogen peroxide $\left(\mathrm{H}_{2} \mathrm{O}_{2}\right)$ and horseradish peroxidase (HRP). Their stiffness was tuned by varying the $\mathrm{H}_{2} \mathrm{O}_{2}$ concentration without changing the amount of polymer precursor. In a 3D context and without any additional biochemical signal, the rate of hMSCs proliferation increased while decreasing the stiffness of the hydrogels. In particular, the cells cultured for 3 weeks in the hydrogel with the lower stiffness expressed much more neuronal markers than those cultured in the stiffer matrices. SCI repair was also attempted in rat models by Hejčl et al. [41] that studied whether it was feasible to bridge a chronic lesion by implanting a hydrogel based on 2-hydroxypropyl methacrylamide functionalized with RGD (Arg-Gly-Asp) sequences either alone or seeded with MSCs. With respect to the control group, the results showed an improvement for the rats implanted with the MSC-laden hydrogels; furthermore, in this case tissue atrophy was prevented, the hydrogels were infiltrated with axons myelinated with Schwann cells, and MSCs were still present in the hydrogels 5 months after implantation. A slight tendency to improvement was also observed for the rats implanted with the hydrogel alone, but it was not statistically significant. Taken together, these results support the therapeutic potential of synergistic approaches integrating MSCs and suitable hydrogels.

A more complex composite material was developed by Park et al. [42], again for the SCI. Their purpose was to test a hyaluronic acid-based hydrogel as a 3D biomimetic scaffold for peptides and growth factors and test this approach in vivo. To provide guidance cues for nerve regeneration, they used a matrix metalloproteinase peptide cross-linker, an IKVAV (Ile-Lys-Val-Ala-Val) peptide derived from laminin and brain-derived neurotrophic factor (BDNF). Human MSCs were embedded in the hydrogels and their neuronal differentiation was induced after culturing in vitro for 10 days. The hydrogels were then injected in vivo into the intrathecal space and the animals were monitored for 6 weeks. If compared to the other tested groups, the rats injected with BDNF-containing materials showed the greatest improvement on locomotive tests during the initial stage after injury.

An important field where NPs might play a key role is MSCs tracking. In fact, the fate of injected or transplanted MSCs into the body in human or animal models is a matter of great debate [43]. To this respect, Zeng et al. [44] investigated the cytotoxicity of superparamagnetic iron oxide nanoparticles (SPIONPs), used as a contrast agent in magnetic resonance imaging (MRI) for labeling cells in vitro and tracking labeled cells after transplantation in vivo, on the neural differentiation of human amniotic membrane-derived 


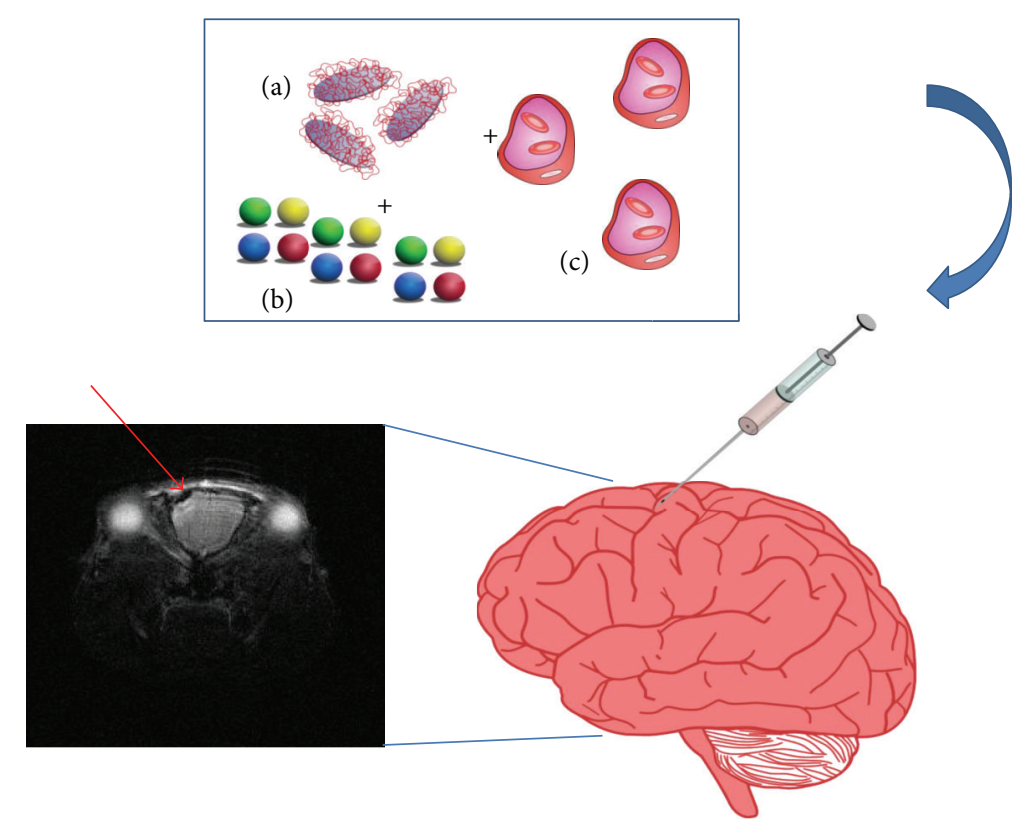

FIGURE 1: Schematic summarizing a possible application of nanostructured gels in the brain. The nanostructured device, composed of (a) an injectable, biocompatible, and resorbable hydrogel, is mixed with a nontoxic amount of NPs (b) and loaded with MSCs (c) that are able to naturally phagocyte the NPs. The device is injected into its target site and the resulting labeled MSCs might be imaged by MRI or other noninvasive techniques. The insert shows an MRI scan of a mouse brain, where the dark spot indicated by the arrow is a denser signal due to SPIONPs.

MSCs (hAM-dMSCs). They found that a single SPIONPlabeled method provided appropriate viability for the labeled hAM-dMSCs and facilitated their evaluation by MRI after transplantation. Another similar study investigated the effective concentration of SPIONPs to track MSCs by evaluating the influence and toxicity of their labeling on multiple differentiated MSCs. The results indicated that the cells were effectively labeled at low concentrations of SPIO and in this condition they maintained their proliferative features and differentiation capacity [45].

\section{MSCs in Parkinson's and Alzheimer's Diseases}

Several data have already outlined that MSCs might play a role in $\mathrm{AD} / \mathrm{PD}$ regenerative or neuroprotective approaches. In fact, MSCs produce a variable panel of protective soluble factors that might be released from a nanostructured hydrogel to the brain parenchyma. For instance, a recent paper by Lee et al. [46] indicated that bone marrow (BM) stem cells showed a beneficial effect through endogenous microglia activation in the brains of $\mathrm{AD}$ mice thanks to the selective production of the chemoattractant factor CCL5. Another group reported that soluble intracellular adhesion molecule1 , secreted by human umbilical cord blood-derived MSCs, reduced amyloid- $\beta$ plaques both in vitro and in vivo, probably thanks to the activation of an amyloid degrading enzyme [47].

In the field of PD, Whone et al. [48] investigated the paracrine effect of MSCs, focusing on the release of glial-derived neurotrophic factor (GDNF). They investigated the neuroprotective properties of unmodified hMSCs on rat catecholaminergic and serotonergic cell cultures exposed to nitric oxide. They found that the soluble factors produced by native hMSCs, requiring no direct cell-cell contact, gave protection not only to cultured monoaminergic perikarya, but also to monoamine neurotransmitter transporter function, and that these beneficial effects were mediated in part by GDNF release.

All these data support the research in the field of nanocomposites and their application for complex disorders like $\mathrm{AD} / \mathrm{PD}$. In this field, the main challenges are the target tissue (brain) that is hardly accessible and the chronic feature of the disorders, requiring prolonged therapies over time (Figure 1).

\section{Conclusions and Perspectives}

In summary, several hydrogel formulations have been specifically developed to mimic 3D scaffolds or nanostructured devices for the release of trophic factors or peptides favoring MSCs adhesion, differentiation into the neuronal lineage, and survival. Considering that NPs are also very promising tools for MSC tracking and taking advantage from the natural phagocytic activity of MSCs, an easy working hypothesis might be the development of a composite gel, nanostructured to label the released or embedded MSCs. This approach might increase the labeling efficiency and decrease possible toxic effects mediated by the NPs, thanks to the copresence of trophic factors. A general comment is that it is mandatory to improve surgical and delivery strategies, as the nervous tissue, and the brain tissue in particular, sets problems of reachability without relevant side effects (unwanted neuronal 

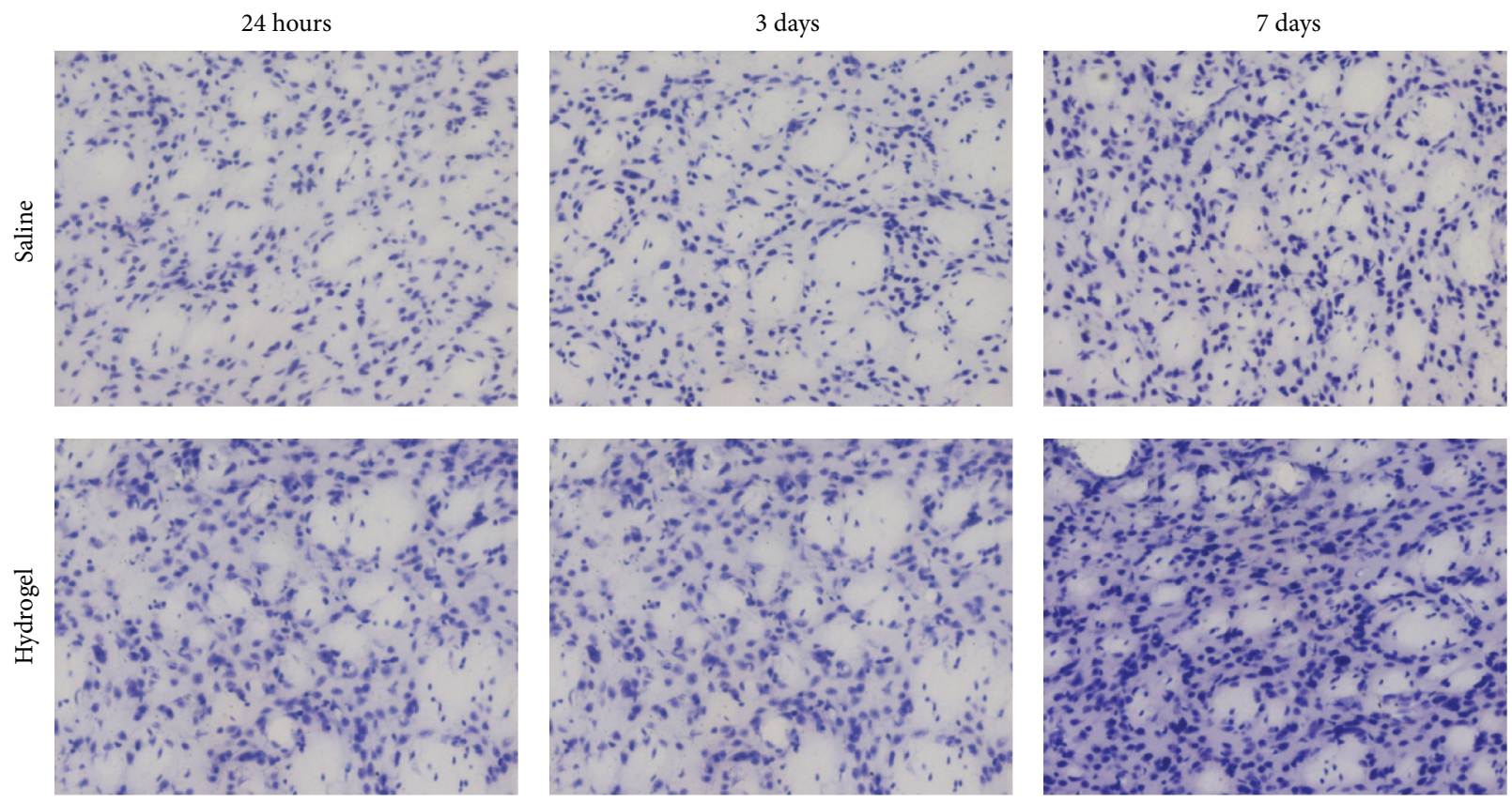

(a)
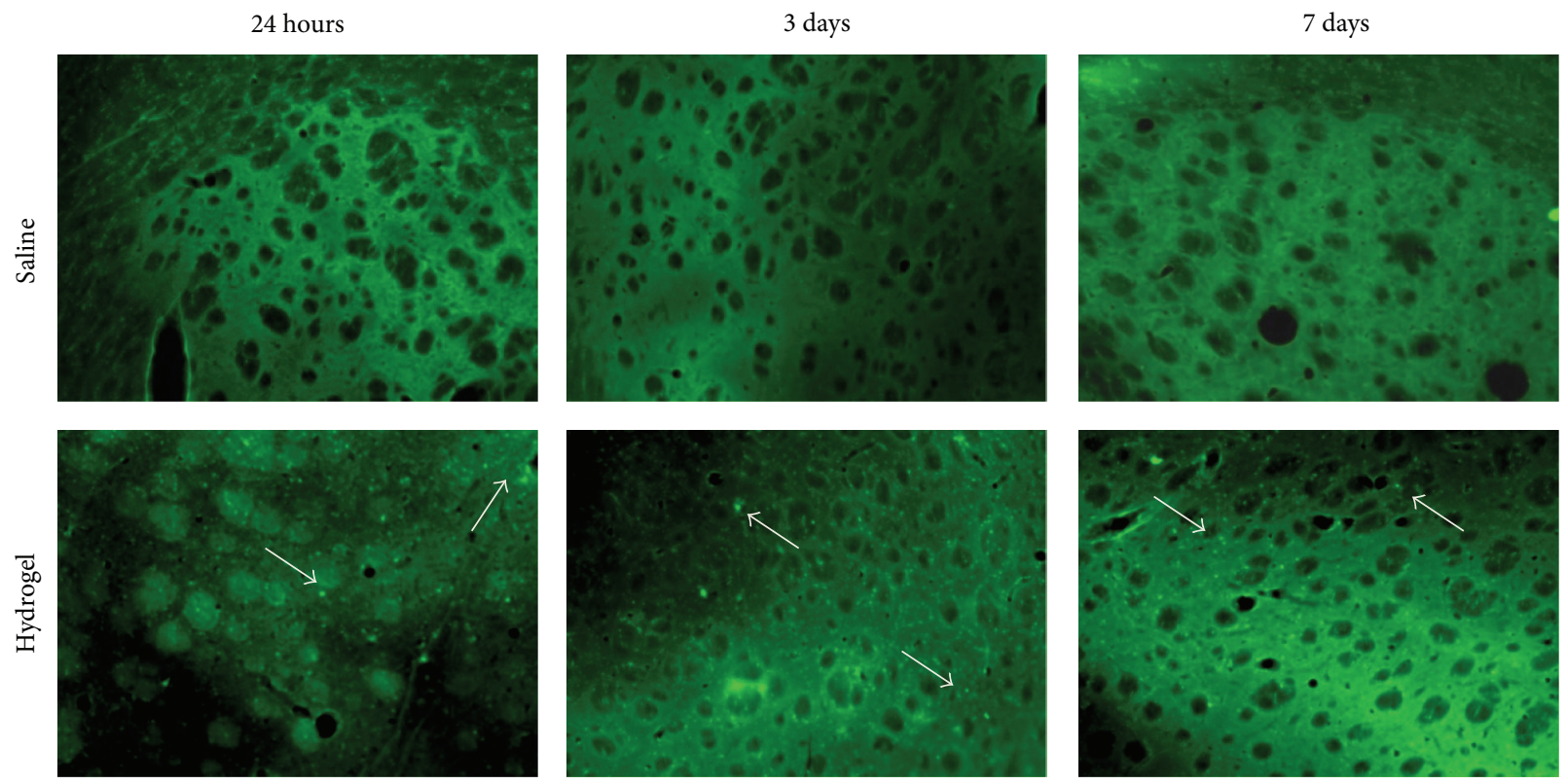

(b)

FIGURE 2: Evaluation of the biocompatibility of an injectable hydrogel formulation for brain applications. We designed and prepared in saline $(0.9 \% \mathrm{NaCl})$ a hydrogel $(\mathrm{pH} 7.2)$ composed of Carbomer $974 \mathrm{P}(4.3 \% \mathrm{w} / \mathrm{v}), \mathrm{PEG}\left(\mathrm{Mw}=600 \mathrm{~g} \mathrm{~mol}^{-1}, 12.2 \% \mathrm{v} / \mathrm{v}\right)$, and glycerol $(14.6 \% \mathrm{v} / \mathrm{v})$. An aliquot $(2 \mu \mathrm{L})$ was diluted $1: 1(\mathrm{v} / \mathrm{v})$ with saline to simulate the cell loading and stereotaxically injected into the mouse striatum, keeping the contralateral brain hemisphere as a control. (a) Nissl staining to point out brain tissue cytoarchitecture. In the hydrogel-injected hemisphere, tissue hypertrophy was present after 7 days. (b) Fluoro-Jade A staining. This staining allows tracking cell damage points that appear as bright green dots. Differently from the control, where few dots were visible, in the hydrogel-injected hemisphere neuronal and glial damage was detectable as early as $24 \mathrm{~h}$ after injection. The white arrows point to the Fluoro-Jade A positive points.

damage and neuroinflammation). In this field, recent works have mainly focused on SCI, a peripheral and thus more easily addressable pathology from the point of view of hydrogelbased therapies. To this end, a recently described epicortical approach for the release of drugs to the brain after a stroke might also be promising for drug delivery [49].

The authors of this review are currently working on the nanostructuring of semi-interpenetrating polymer networks 


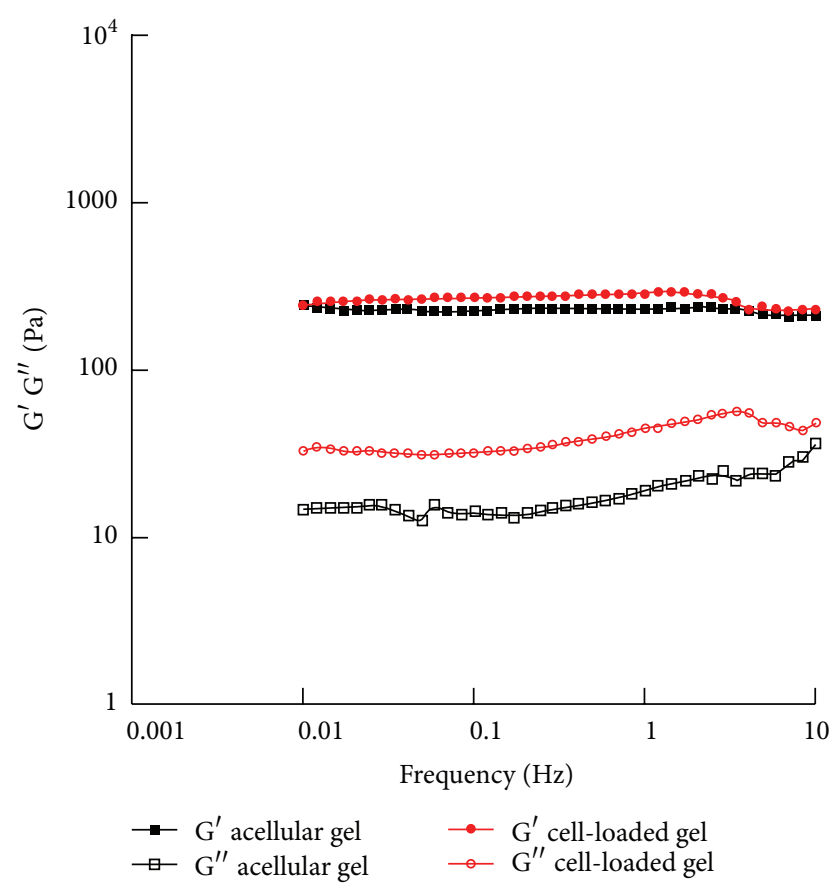

Figure 3: Small amplitude oscillatory shear tests. After the preparation, small amplitude oscillatory shear tests were performed on acellular and cell-laden $\left(0.5 \cdot 10^{6}\right.$ cells $/ \mathrm{mL}$ gel $)$ PEG/Carbomer-based hydrogels at $37^{\circ} \mathrm{C}$ with frequency ranging from 0.01 to $10 \mathrm{~Hz}$. Both acellular and cell-laden materials showed a gel-like behaviour as the storage modulus $G^{\prime}$ was greater than the loss modulus $G^{\prime \prime}$ in the frequency range investigated. In particular, $G^{\prime}$ was almost constant for both acellular gels $(227.5 \pm 34.1 \mathrm{~Pa})$ and cell-laden constructs $(280.0 \pm 41.0 \mathrm{~Pa})$.

based on collagen and PEG, with the specific focus of their optimization for brain delivery that, as outlined, is a critical and unmet improvement for advanced therapies based on biocompatible devices. In particular, we are investigating the effects of NPs in modulating the rheological and functional properties of the hydrogels and also improving the sustained release of therapeutic recombinant proteins (i.e., the chaperone protein Hsp70 that has proved to be beneficial in several models of neurodegeneration) [50-53]. Our view is that nanocomposite gels might be a powerful tool to achieve a controlled and long-lasting drug delivery in close contact with the damaged areas in the case of chronic neurodegeneration, an innovative strategy that might open the way to new therapies [54]. To this purpose, the development of highly biocompatible hydrogel-based matrices is mandatory to avoid unwanted tissue damage or trigger a dangerous inflammatory response in the very peculiar biologic environment represented by the brain nervous tissue. To clearly demonstrate the latter point, in Figure 2 we show a time course of a biocompatibility study to assess the brain cytoarchitecture and inflammatory response after the injection of a hydrogel composed of Carbomer 974P $(2.15 \% \mathrm{w} / \mathrm{v})$, PEG $\left(\mathrm{Mw}=600 \mathrm{~g} \mathrm{~mol}^{-1}, 6.1 \% \mathrm{v} / \mathrm{v}\right)$, and glycerol $(7.3 \% \mathrm{v} / \mathrm{v})$ and prepared in saline ( $\mathrm{pH} 7.2)$. Just after $24 \mathrm{~h}$, we noticed tissue damage and neurodegeneration, while after 7

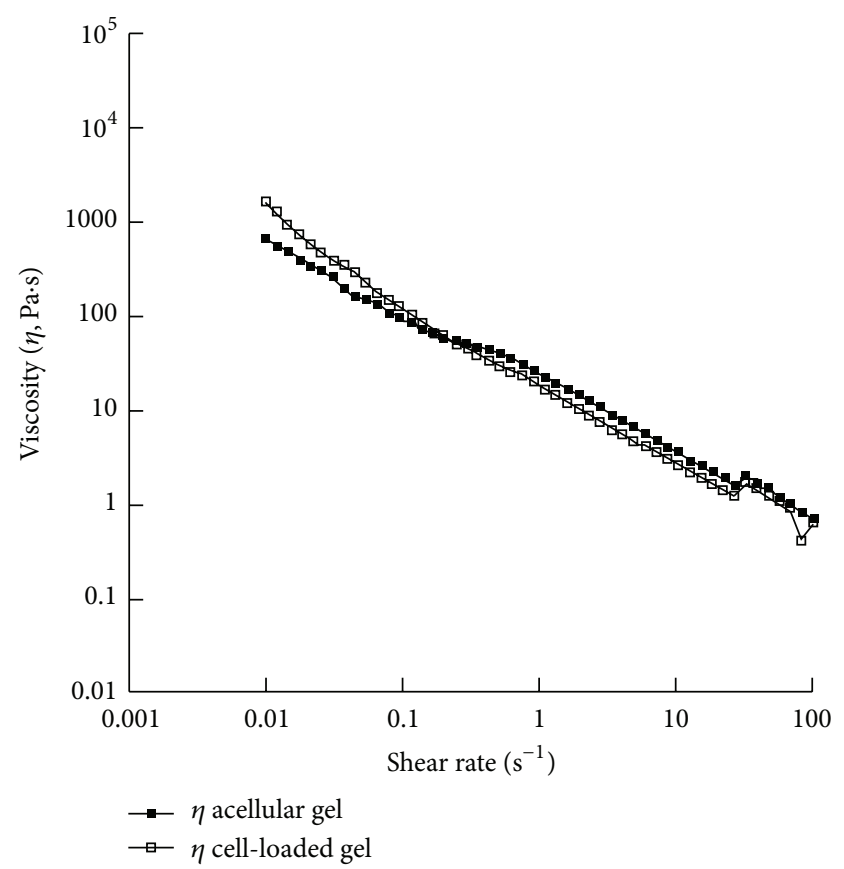

Figure 4: Steady shear tests. After the preparation, steady shear measurements were performed on acellular and cell-laden $\left(0.5 \cdot 10^{6}\right.$ cells $/ \mathrm{mL}$ gel) $\mathrm{PEG} /$ Carbomer-based hydrogels at $37^{\circ} \mathrm{C}$ with shear rate ranging from 0.01 to $100 \mathrm{~s}^{-1}$. In both cases, the viscosity decreased as the shear rate increased (shear thinning behaviour). In particular, when the shear rate increased from 0.01 to $100 \mathrm{~s}^{-1}$, the viscosity decreased from 664.5 to $0.7 \mathrm{~Pa}$.s for acellular gels and from 1608.1 to $0.6 \mathrm{~Pa} \cdot \mathrm{s}$ for cell-laden constructs.

days hypertrophy and neurodegeneration were apparent in the hydrogel-injected hemisphere, suggestive of a negative response to the biomaterial, even though this formulation had suitable rheologic and structural properties. From this example it is apparent that our work, with its focus on brain biocompatibility, is an original step forward in the field.

As highlighted in the first section, viscoelastic features, functional injectability, and transport properties (i.e., permeability), together with biocompatibility and biological response, play a crucial role in driving the performances of hydrogel-based devices. In particular, the embedded cells may strongly change the properties of the starting material and we aimed at assessing this point focusing on MSCs embedding. As an example to clearly evidence this feature, in Figures 3 and 4 we show the results from small amplitude oscillatory shear tests and steady shear measurements for the acellular and cell-laden PEG/Carbomer-based hydrogels. The results indicated that the presence of the cells initially increased the stiffness (as suggested by the fact that $G^{\prime}$ was lower for acellular materials) and the resistance to flow under shear, especially at low shear rates (as shown by the higher viscosity values for cell-laden constructs).

However, to gain a deeper knowledge about the effects of cell loading on the hydrogel properties, these features should be assessed at different time points after cell seeding. Moreover, the evaluation of how the injection through clinical 
catheters (already applied for Parkinson's disease MSC-based experimental therapies [55]) modifies the viscoelastic properties might represent the first step of a systematic analysis for hydrogels and nanocomposites optimization in the view of a clinical application.

\section{Disclosure and Ethical Approval}

The experimental procedures described and involving animals and their care were conducted in conformity with the institutional guidelines at the IRCCS-Istituto di Ricerche Farmacologiche "Mario Negri" in compliance with national (Decreto Legge no. 116/92, Gazzetta Ufficiale, supplement 40, February 18, 1992; Circolare no. 8, Gazzetta Ufficiale, July 14,1994 ) and international laws and policies (EEC Council Directive 86/609, OJL 358, 1, December 12, 1987; Guide for the Care and Use of Laboratory Animals, US National Research Council (eighth edition), 2011).

\section{Conflict of Interests}

The authors declare that there is no conflict of interests regarding the publication of this paper.

\section{Acknowledgments}

The authors gratefully thank Sara Batelli for performing Nissl and Fluoro-Jade A staining. The authors' research work was supported by Fondazione Cariplo (Contract Grant no.: 20110335 to C. Giordano).

\section{References}

[1] R. L. Goya, W. Kuan, and R. A. Barker, "The future of cell therapies in the treatment of Parkinson's disease," Expert Opinion on Biological Therapy, vol. 7, no. 10, pp. 1487-1498, 2007.

[2] N. Payne, C. Siatskas, A. Barnard, and C. C. A. Bernard, "The prospect of stem cells as multi-faceted purveyors of immune modulation, repair and regeneration in multiple sclerosis," Current Stem Cell Research and Therapy, vol. 6, no. 1, pp. 50-62, 2011.

[3] M. Dadon-Nachum, E. Melamed, and D. Offen, "Stem cells treatment for sciatic nerve injury," Expert Opinion on Biological Therapy, vol. 11, no. 12, pp. 1591-1597, 2011.

[4] C. Giordano, D. Albani, A. Gloria et al., "Multidisciplinary perspectives for Alzheimer's and Parkinson's diseases: hydrogels for protein delivery and cell-based drug delivery as therapeutic strategies," International Journal of Artificial Organs, vol. 32, no. 12, pp. 836-850, 2009.

[5] C. Giordano, D. Albani, A. Gloria et al., "Nanocomposites for neurodegenerative diseases: hydrogel-nanoparticle combinations for a challenging drug delivery," International Journal of Artificial Organs, vol. 34, no. 12, pp. 1115-1127, 2011.

[6] F. Munarin, P. Petrini, S. Bozzini, and M. C. Tanzi, "New perspectives in cell delivery systems for tissue regeneration: natural-derived injectable hydrogels," Journal of Applied Biomaterials \& Functional Materials, vol. 10, no. 2, pp. 67-81, 2012.

[7] L. Tartivel, M. Behl, M. Schroeter, and A. Lendlein, "Hydrogel networks based on ABA triblock copolymers," Journal of
Applied Biomaterials \& Functional Materials, vol. 10, no. 3, pp. 243-248, 2012.

[8] V. Cirillo, V. Guarino, and L. Ambrosio, "Design of bioactive electrospun scaffolds for bone tissue engineering," Journal of Applied Biomaterials \& Functional Materials, vol. 10, no. 3, pp. 223-228, 2012.

[9] L. Pescosolido, S. Miatto, C. Di Meo et al., "Injectable and in situ gelling hydrogels for modified protein release," European Biophysics Journal, vol. 39, no. 6, pp. 903-909, 2010.

[10] X. Li, E. Katsanevakis, X. Liu, N. Zhang, and X. Wen, "Engineering neural stem cell fates with hydrogel design for central nervous system regeneration," Progress in Polymer Science, vol. 37, no. 8, pp. 1105-1129, 2012.

[11] A. J. Michalek, M. R. Buckley, L. J. Bonassar, I. Cohen, and J. C. Iatridis, "The effects of needle puncture injury on microscale shear strain in the intervertebral disc annulus fibrosus," Spine Journal, vol. 10, no. 12, pp. 1098-1105, 2010.

[12] J. T. Martin, D. J. Gorth, E. E. Beattie, B. D. Harfe, L. J. Smith, and D. M. Elliott, "Needle puncture injury causes acute and longterm mechanical deficiency in a mouse model of intervertebral disc degeneration," Journal of Orthopaedic Research, vol. 31, no. 8, pp. 1276-1282, 2013.

[13] K. Singh, K. Masuda, and H. S. An, "Animal models for human disc degeneration," Spine Journal, vol. 5, supplement, no. 6, pp. 267S-279S, 2005.

[14] J. Silva-Correia, V. Miranda-Gonçalves, A. J. Salgado et al., "Angiogenic potential of gellan-gum-based hydrogels for application in nucleus pulposus regeneration: in vivo study," Tissue Engineering A, vol. 18, no. 11-12, pp. 1203-1212, 2012.

[15] K. Benz, C. Stippich, C. Osswald et al., "Rheological and biological properties of a hydrogel support for cells intended for intervertebral disc repair," BMC Musculoskeletal Disorders, vol. 13, article 54, 2012.

[16] S. Reitmaier, U. Wolfram, A. Ignatius et al., "Hydrogels for nucleus replacement: facing the biomechanical challenge," Journal of the Mechanical Behavior of Biomedical Materials, vol. 14, pp. 67-77, 2012.

[17] K. A. Jansen, R. G. Bacabac, I. K. Piechocka, and G. H. Koenderink, "Cells actively stiffen fibrin networks by generating contractile stress," Biophysical Journal, vol. 105, no. 10, pp. 22402251, 2013.

[18] P. A. Galie and J. P. Stegemann, "Simultaneous application of interstitial flow and cyclic mechanical strain to a three-dimensional cell-seeded hydrogel," Tissue Engineering C, vol. 17, no. 5, pp. 527-536, 2011.

[19] D. Kesselman, O. Kossover, I. Mironi-Harpaz, and D. Seliktar, "Time-dependent cellular morphogenesis and matrix stiffening in proteolytically responsive hydrogels," Acta Biomaterials, vol. 9, no. 8, pp. 7630-7639, 2013.

[20] J. Silva-Correia, A. Gloria, M. B. Oliveira et al., "Rheological and mechanical properties of acellular and cell-laden methacrylated gellan gum hydrogels," Journal of Biomedical Materials Research A, vol. 101, no. 12, pp. 3438-3446, 2013.

[21] T. Russo, U. D’Amora, A. Gloria et al., "Systematic analysis of injectable materials and 3D rapid prototyped magnetic scaffolds: from CNS applications to soft and hard tissue repair/ regeneration," Procedia Engineering, vol. 59, pp. 233-239, 2013.

[22] A. Gloria, A. Borzacchiello, F. Causa, and L. Ambrosio, "Rheological characterization of hyaluronic acid derivatives as injectable materials toward nucleus pulposus regeneration," Journal of Biomaterials Applications, vol. 26, no. 6, pp. 745-759, 2012. 
[23] L. Nicolais, A. Gloria, and L. Ambrosio, "The mechanics of biocomposites," in Biomedical Composites, L. Ambrosio, Ed., pp. 411-440, CRC Press, London, UK, 2010.

[24] H. Fischer, "Polymer nanocomposites: from fundamental research to specific applications," Materials Science and Engineering C, vol. 23, no. 6-8, pp. 763-772, 2003.

[25] A. Borzacchiello, A. Gloria, L. Mayol et al., "Natural/synthetic porous scaffold designs and properties for fibro-cartilaginous tissue engineering," Journal of Bioactive and Compatible Polymers, vol. 26, no. 5, pp. 437-451, 2011.

[26] M. F. Koudehi, A. A. Fooladi, K. Mansoori, Z. Jamalpoor, A. Amiri, and M. R. Nourani, "Preparation and evaluation of novel nano-bioglass/gelatin conduit for peripheral nerve regeneration," Journal of Materials Science, 2013.

[27] M. Rides, C. R. G. Allen, and G. D. Sims, "Rheological characterisation of a highly filled curing system using a disposable extrusion rheometer," Polymer Testing, vol. 29, no. 2, pp. 164$169,2010$.

[28] A. A. Collyer and D. W. Clegg, Rheological Measurement, Chapman \& Hall, London, UK, 1998.

[29] G. A. Ateshian, W. H. Warden, J. J. Kim, R. P. Grelsamer, and V. C. Mow, "Finite deformation biphasic material properties of bovine articular cartilage from confined compression experiments," Journal of Biomechanics, vol. 30, no. 11-12, pp. 1157-1164, 1997.

[30] A. K. Williamson, A. C. Chen, and R. L. Sah, "Compressive properties and function-composition relationships of developing bovine articular cartilage," Journal of Orthopaedic Research, vol. 19, no. 6, pp. 1113-1121, 2001.

[31] M. H. Holmes and V. C. Mow, "The nonlinear characteristics of soft gels and hydrated connective tissus in ultrafiltration," Journal of Biomechanics, vol. 23, no. 11, pp. 1145-1156, 1990.

[32] U. Freudenberg, A. Hermann, P. B. Welzel et al., "A star-PEGheparin hydrogel platform to aid cell replacement therapies for neurodegenerative diseases," Biomaterials, vol. 30, no. 28, pp. 5049-5060, 2009.

[33] R. Mooney, S. Haeger, R. Lawal et al., "Control of neural cell composition in poly(ethylene glycol) hydrogel culture with soluble factors," Tissue Engineering A, vol. 17, no. 21-22, pp. 28052815, 2011.

[34] Z. Zhou, P. Yu, H. M. Geller, and C. K. Ober, "The role of hydrogels with tethered acetylcholine functionality on the adhesion and viability of hippocampal neurons and glial cells," Biomaterials, vol. 33, no. 8, pp. 2473-2481, 2012.

[35] O. Lindvall, Z. Kokaia, and A. Martinez-Serrano, "Stem cell therapy for human neurodegenerative disorders-how to make it work," Nature Medicine, vol. 10, supplement, pp. S42-S50, 2004.

[36] M. Ghoreishian, M. Rezaei, B. H. Beni, S. H. Javanmard, B. M. Attar, and H. Zalzali, "Facial nerve repair with Gore-Tex tube and adipose-derived stem cells: an animal study in dogs," Journal of Oral and Maxillofacial Surgery, vol. 71, no. 3, pp. 577587, 2013.

[37] A. Š. Kubinová, D. Horák, A. Hejčl, Z. Plichta, J. Kotek, and E. Syková, "Highly superporous cholesterol-modified poly(2hydroxyethyl methacrylate) scaffolds for spinal cord injury repair," Journal of Biomedical Materials Research A, vol. 99, no. 4, pp. 618-629, 2011.

[38] G. Perale, C. Giordano, F. Bianco et al., "Hydrogel for cell housing in the brain and in the spinal cord," International Journal of Artificial Organs, vol. 34, no. 3, pp. 295-303, 2011.
[39] H. Gu, Z. Yue, W. S. Leong, B. Nugraha, and L. P. Tan, “Control of in vitro neural differentiation of mesenchymal stem cells in 3D macroporous, cellulosic hydrogels," Regenerative Medicine, vol. 5, no. 2, pp. 245-253, 2010.

[40] L. Wang, J. E. Chung, P. Chan, and M. Kurisawa, "Injectable biodegradable hydrogels with tunable mechanical properties for the stimulation of neurogenesic differentiation of human mesenchymal stem cells in 3D culture," Biomaterials, vol. 31, no. 6, pp. 1148-1157, 2010.

[41] A. Hejčl, J. Šedý, M. Kapcalová et al., "HPMA-RGD hydrogels seeded with mesenchymal stem cells improve functional outcome in chronic spinal cord injury," Stem Cells and Development, vol. 19, no. 10, pp. 1535-1546, 2010.

[42] J. Park, E. Lim, S. Back, H. Na, Y. Park, and K. Sun, "Nerve regeneration following spinal cord injury using matrix metalloproteinase-sensitive, hyaluronic acid-based biomimetic hydrogel scaffold containing brain-derived neurotrophic factor," Journal of Biomedical Materials Research A, vol. 93, no. 3, pp. 1091-1099, 2010.

[43] E. Sykové and P. Jendelová, "Migration, fate and in vivo imaging of adult stem cells in the CNS," Cell Death and Differentiation, vol. 14, no. 7, pp. 1336-1342, 2007.

[44] G. Zeng, G. Wang, F. Guan et al., "Human amniotic membranederived mesenchymal stem cells labeled with superparamagnetic iron oxide nanoparticles: the effect on neuron-like differentiation in vitro," Molecular and Cellular Biochemistry, vol. 357, no. 1-2, pp. 331-341, 2011.

[45] K. L. Jae, K. L. Man, J. J. Hye et al., "Efficient intracytoplasmic labeling of human umbilical cord blood mesenchymal stromal cells with ferumoxides," Cell Transplantation, vol. 16, no. 8, pp. 849-857, 2007.

[46] J. K. Lee, E. H. Schuchman, H. K. Jin, and J. S. Bae, "Soluble CCL5 derived from bone marrow-derived mesenchymal stem cells and activated by amyloid $\beta$ ameliorates Alzheimer's disease in mice by recruiting bone marrow-induced microglia immune responses," Stem Cells, vol. 30, no. 7, pp. 1544-1555, 2012.

[47] J.-Y. Kim, D. H. Kim, J. H. Kim et al., "Soluble intracellular adhesion molecule-1 secreted by human umbilical cord bloodderived mesenchymal stem cell reduces amyloid-B plaques," Cell Death and Differentiation, vol. 19, no. 4, pp. 680-691, 2012.

[48] A. L. Whone, K. Kemp, M. Sun, A. Wilkins, and N. J. Scolding, "Human bone marrow mesenchymal stem cells protect catecholaminergic and serotonergic neuronal perikarya and transporter function from oxidative stress by the secretion of glial-derived neurotrophic factor," Brain Research, vol. 1431, pp. 86-96, 2012.

[49] M. J. Caicco, M. J. Cooke, Y. Wang, A. Tuladhar, C. M. Morshead, and M. S. Shoichet, "A hydrogel composite system for sustained epi-cortical delivery of Cyclosporin A to the brain for treatment of stroke," Journal of Controlled Release, vol. 166, no. 3, pp. 197-202, 2013.

[50] D. Albani, E. Peverelli, R. Rametta et al., "Protective effect of TAT-delivered $\alpha$-synuclein: relevance of the C-terminal domain and involvement of HSP70," The FASEB Journal, vol. 18, no. 14, pp. 1713-1715, 2004.

[51] S. Batelli, D. Albani, R. Rametta et al., "DJ-1 modulates $\alpha$ synuclein aggregation state in a cellular model of oxidative stress: relevance for Parkinson's disease and involvement of HSP70," PLoS ONE, vol. 3, no. 4, Article ID e1884, 2008.

[52] U. K. Jinwal, E. Akoury, J. F. Abisambra et al., "Imbalance of Hsp70 family variants fosters tau accumulation," The FASEB Journal, vol. 27, no. 4, pp. 1450-1459, 2013. 
[53] J. Cui, Y. Wang, Q. Dong et al., "Morphine protects against intracellular amyloid toxicity by inducing estradiol release and upregulation of Hsp70," Journal of Neuroscience, vol. 31, no. 45, pp. 16227-16240, 2011.

[54] F. Baino, S. Fiorilli, R. Mortera et al., "Mesoporous bioactive glass as a multifunctional system for bone regeneration and controlled drug release," Journal of Applied Biomaterials \& Functional Materials, vol. 1, no. 10, pp. 12-21, 2012.

[55] N. K. Venkataramana, R. Pal, S. A. V. Rao et al., "Bilateral transplantation of allogenic adult human bone marrow-derived mesenchymal stem cells into the subventricular zone of Parkinson's disease: a pilot clinical study," Stem Cells International, vol. 2012, Article ID 931902, 12 pages, 2012. 

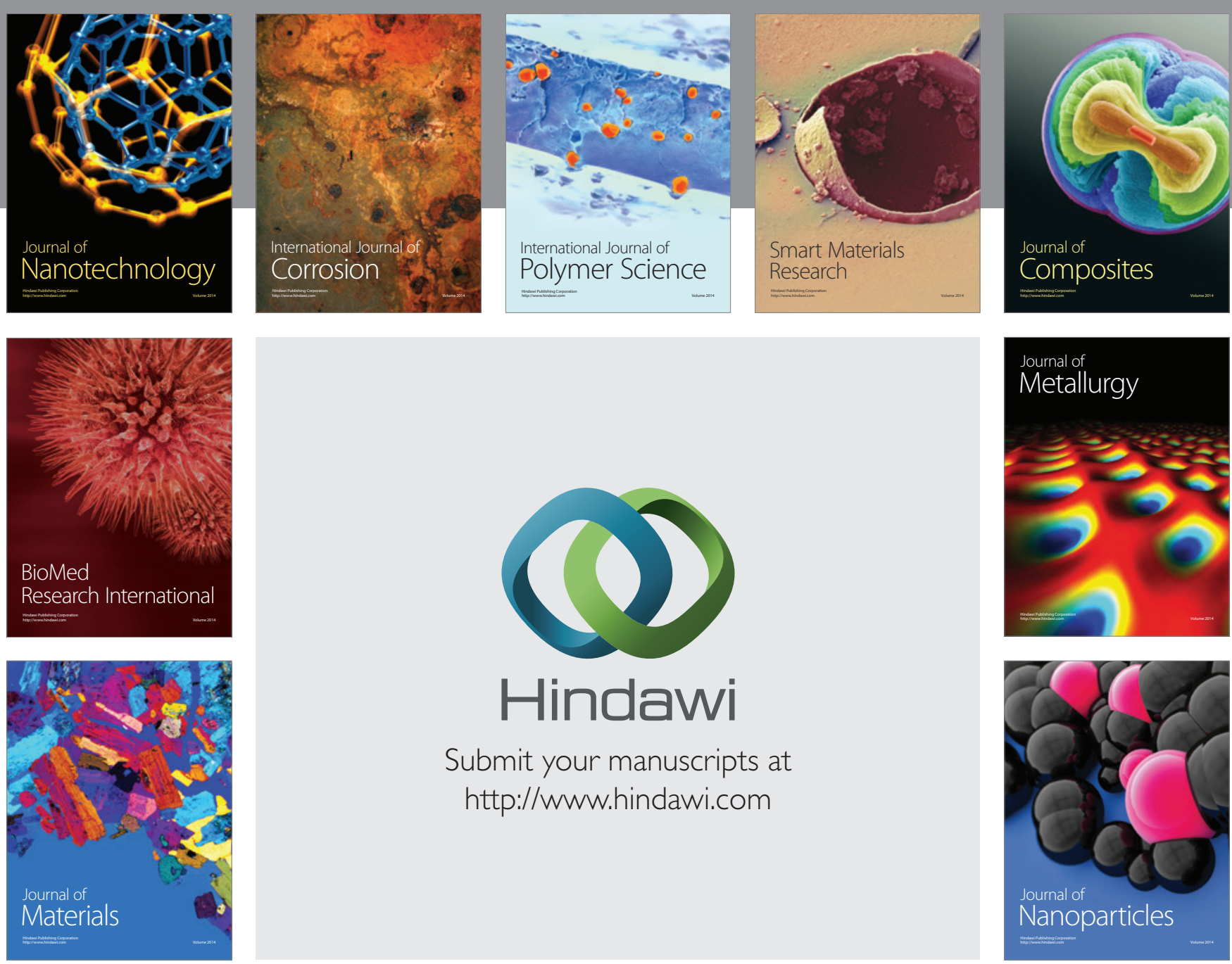

Submit your manuscripts at http://www.hindawi.com
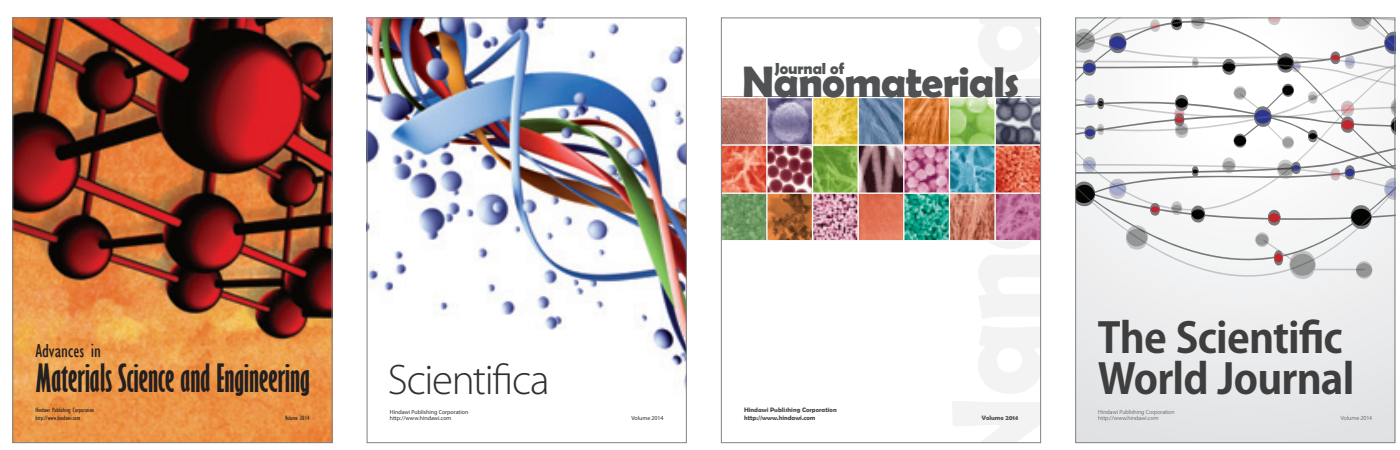

\section{The Scientific World Journal}
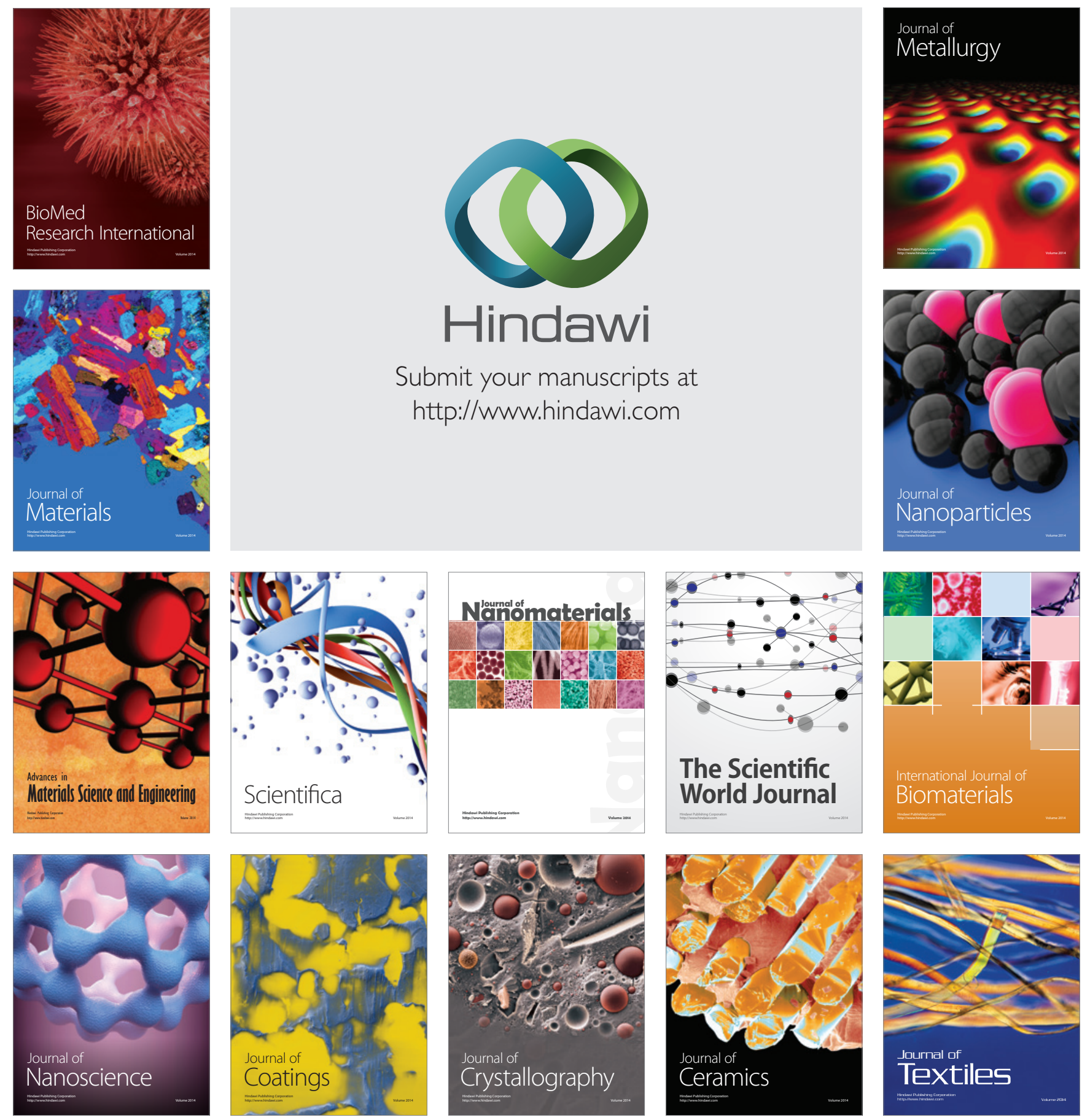